\title{
A Study on the Integrated MEMS Gyroscope Array for Improving the Accuracy of Stabilization Precision in Small GIMBAL
}

\author{
Him-Chan Cho ${ }^{1}$, Dong-Gi Kwag ${ }^{2 *}$ \\ 1 Researcher, Department of Aeronautical Systems Engineering, Hanseo University, Gomseom-ro 236- \\ 49 Nam-Myeon Taean-Gun Chuncheongnam-do,32158, Korea \\ $2 *$ Professor, Department of Aeromechanical Engineering, Hanseo University, Gomseom-ro 236-49 Nam- \\ Myeon Taean-Gun Chuncheongnam-do,32158, Korea \\ eunsroom3@naver.com ${ }^{1}$,dgkwag@hanseo.ac.kr*²
}

Article History:Received:11 november 2020; Accepted: 27 December 2020; Published online: 05 April 2021

\begin{abstract}
The output values Gyroscope measures will contain Bias, so errors gradually accumulate. Considering this point, a study on multi-sensors was conducted. An analysis of Gyroscope noise characteristics was carried out, and the modeling for Kalman Filter was performed based on the analysis. Afterwards, data values were extracted and analyzed through an experiment. Gyroscope's Angle Random Walk and Rate Random Walk were derived using Allan Variance, and based on this, Kalman Filter covariance matrix was formed. Data reception algorithms were constructed using Matlab Simulink, and an experiment was conducted using MicroLabBox and Rate Table. The final research objective is to apply the results of this study to 2-Axis Small Gimbal to improve stabilization precision.
\end{abstract}

Keywords: Multi-Sensors, Noise Characteristics, Kalman Filter, Allan Variance.

\section{Introduction}

With the advancement of semiconductor technology, research and development for inexpensive, low power-consuming, and small inertial sensors based on MEMS technology are being carried out. MEMS (Micro-Electro Mechanical System) IMU (Inertial Measurement Unit) sensors are used in various areas such as aircraft, inertial navigation, and platform stabilization [1]. MEMS IMU sensor refers to a sensor with Gyroscope, accelerometer, magnetometer and altimeter. Bias from Gyroscope output gradually accumulates errors causing Drift, which significantly lowers the performance of the sensors. The use of multi-sensors can compensate for the defect of a single sensor to improve sensor accuracy. In order to improve the accuracy of the information about the motion of an object, the method of using multi-sensors instead of a single sensor has already been used in many engineering fields ranging from medical systems to integrated navigation systems [2]. In 1992, Weis and Allan introduced a high-precise watch named 'Smart Watch' with an error of 1 second by combining three inexpensive wrist watches [3]. This technology actually used heterogeneous sensor data fusion to improve accuracy. For higher accuracy of MEMS Gyroscope, researchers have recently conducted a study similar to sensor data fusion, and Bayard created a highly accurate virtual sensor by combining inexpensive MEMS Gyroscope. This technology was called 'Virtual Gyroscope' which was designed to have improved accuracy by using a homogeneous sensor unlike Smart Watch [4]. In order to use multiple MEMS IMUs, KF (Kalman Filter) is typically used to build a system. For the application of KF, it needs to obtain information on the noise state and system variables to determine the noise characteristics of sensors. This study aims to improve the accuracy by identifying the noise characteristics of a target sensor and combining two low-cost Gyroscopes, so as to improve the stabilization accuracy of a 2-Axis Small Gimbal. And this is the final objective of this study. Fig 1 shows the appearance of a Small Gimbal, and Fig 2 shows the diagram of Gyroscope array.
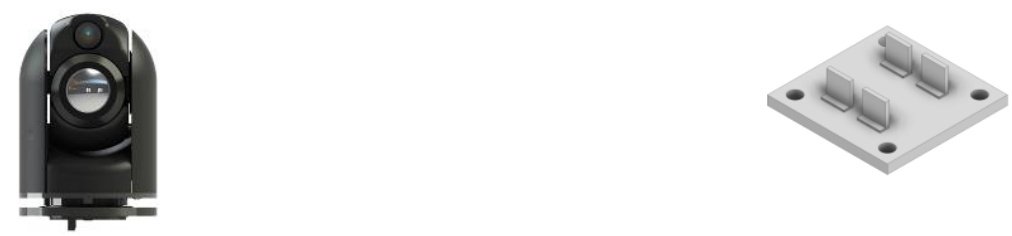

Fig 1. 2-Axis Small Gimbal

Fig 2. Schematic of Gyroscope array

\section{Research Method}

The purpose of this study was to build a multi-sensor system for the application to a 2-Axis Small Gimbal. As a KF-based study, it constructed the overall system by selecting a mathematical model for multi-

*Corresponding author: Dong-Gi Kwag

Professor, Department of Aeromechanical Engineering, Hanseo University,

Gomseom-ro 236-49 Nam-Myeon Taean-Gun Chuncheongnam-do,32158, Korea

dgkwag@hanseo.ac.kr 
sensor fusion and identified the noise characteristics of the target Gyroscope through the analysis of AV (Allan Variance) and PSD (Power Spectral Density). An experiment was conducted using MicroLabBox and Rate Table.

\subsection{Mathematical Model for Multi-sensor Fusion}

\subsubsection{Kalman Filter-based Fusion Method}

Multi-sensor data fusion is a technology that enables combining information from several data in order to form a unified data. And it is currently used in many fields such as robotics, image and image processing, sensor network, and intelligent system design. Multi-sensor data fusion has been mainly used in military applications over the past decades, but recently it has been also widely used in civilian applications [5]. Kalman Filter has been extensively studied as a filter for data fusion, and it can be largely divided into MF(Measurement Fusion) and TTF(Track to Track Fusion) according to the fusion stage for efficient data management, as shown in Fig 3 and Fig 4[6]. In this study, the principle of MEMS Gyroscope array multisignal fusion is shown in Fig 5, and Kalman Filter was used to derive the optimal estimate of angular velocity signals and Drift errors by fusing multi-signal velocities.

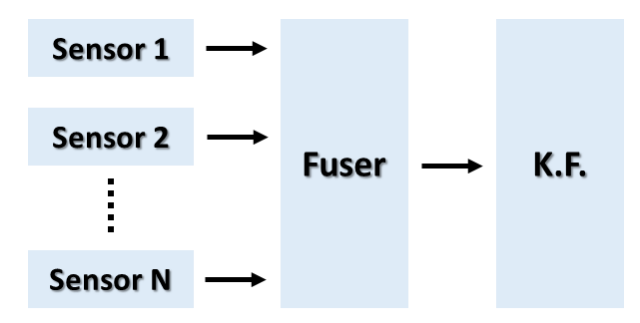

Fig 3. Measurement Fusion

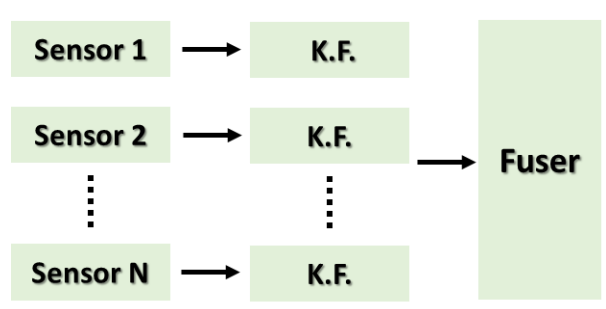

Fig 4. Track to Track Fusion

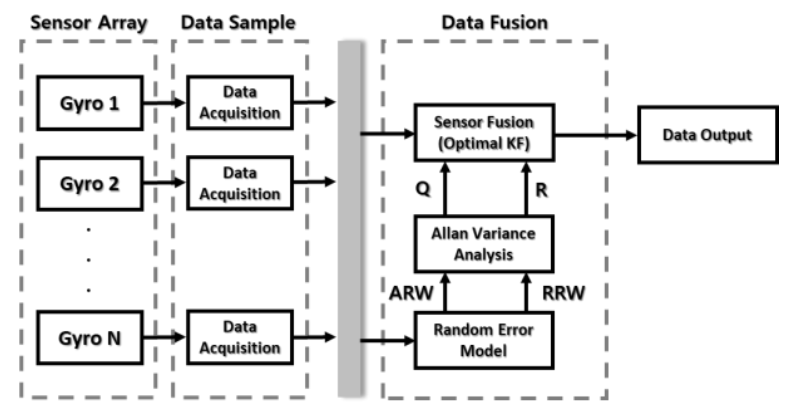

Fig 5. Principle of the Multiple Rate Signals Fusion of a MEMS Gyroscope Array

\subsection{IMU Noise Characteristics Analysis}

\subsubsection{Random Noise Modeling for MEMS Gyroscope}

IMU signals are output including various noises that cause large errors in position, speed, and posture. In order to predict the accurate performance of an inertial system, it begins with appropriate noise modeling for sensors, and the noise characteristics of sensors can be derived through AV (Allan Variance) [7]. Most of the inertial sensor noises are ARW (Angle Random Walk) and RRW (Rate Random Walk), and additionally there is BI (Bias Instability) [8]. Fig 6 shows Random Error Model, and the noise model is shown in Table 1. Equation (1) is an AV Gyroscope measurement model.

Table 1. Noise Model 


\begin{tabular}{|c|c|c|}
\hline Category & Symbol & Definition \\
\hline $\begin{array}{c}\text { ARW(Angle Random } \\
\text { Walk) }\end{array}$ & $\mathbf{N}$ & White noise, short-term high frequency noise \\
\hline $\begin{array}{c}\text { BI(Bias Instability) } \\
\text { RRW(Rate Random } \\
\text { Walk) }\end{array}$ & $\mathbf{B}$ & $\begin{array}{c}\text { Flicker noise found in the low frequency, and a noise important } \\
\text { for determining sensor stability }\end{array}$ \\
\hline \multicolumn{2}{|r|}{$\Omega(t)=\Omega_{\text {Ideal }}(t)+\operatorname{Bias}_{N}(t)+\operatorname{Bias}_{B}(t)+\operatorname{Bias}_{K}(t) \quad(1)$} \\
\hline
\end{tabular}

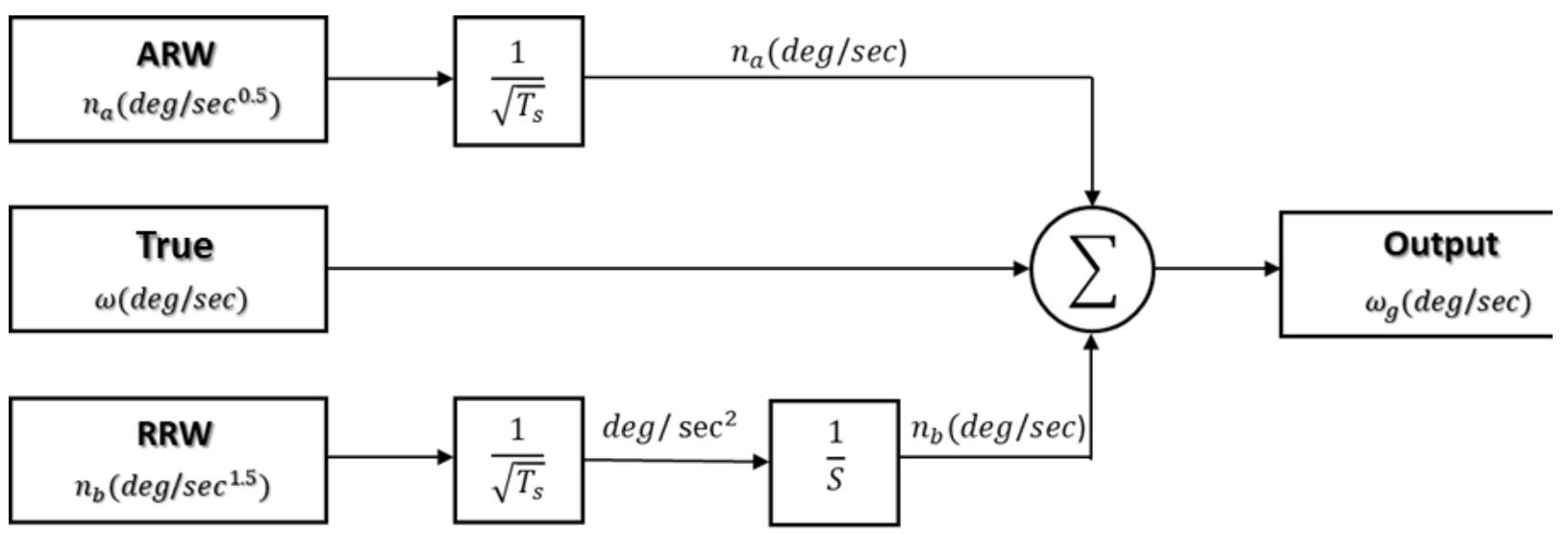

Fig 6. Random error model of MEMS gyroscope

\subsubsection{Calculation of Allan Variance}

PSD (Power Spectral Density) and AV (Allan Variance) techniques were used to understand the characteristics of Random Noise. In this study, AV technique was performed to identify measurement noise in IMU signal, and PSD technique was used to secure data reliability [9].

\subsubsection{Allan Variance Calculation}

The angular velocity of Gyroscope was sampled with M sample(s) at a sampling time of $\boldsymbol{\tau}_{\boldsymbol{s}}$, and then $\boldsymbol{L}(\boldsymbol{M}<$ $\frac{\boldsymbol{L}-\mathbf{1}}{\mathbf{2}}$ ) data sample(s). Each group is called Cluster and has data corresponding to, $\boldsymbol{\tau}=\boldsymbol{L} \boldsymbol{\tau}_{\boldsymbol{s}}$. When the continuous output of Gyroscope is set to $\boldsymbol{\Omega}(\boldsymbol{t})$ and measured at discrete time intervals of $\boldsymbol{t}=\boldsymbol{k} \boldsymbol{\tau}_{\boldsymbol{s}}(\mathrm{k}=1,2,3 \ldots \mathrm{M})$, if the angle is indicated as $\boldsymbol{\theta}_{\boldsymbol{k}}\left(\boldsymbol{\theta}(\boldsymbol{t})=\boldsymbol{\theta}\left(\boldsymbol{k} \boldsymbol{\tau}_{\boldsymbol{s}}\right)=\boldsymbol{\theta}_{\boldsymbol{k}}\right)$, the average angle between $\boldsymbol{k} \boldsymbol{\tau}_{\boldsymbol{s}}$ and $\boldsymbol{k} \boldsymbol{\tau}_{\boldsymbol{s}}+\boldsymbol{\tau}$ is shown as Equation (3).

$$
\begin{array}{r}
\theta(t)=\int_{0}^{t} \Omega\left(t^{\prime}\right) d t^{\prime} \\
\bar{\theta}_{k}(\tau)=\frac{1}{\tau} \int_{k \tau_{s}}^{k \tau_{s}+t} \Omega(t) d t
\end{array}
$$

AV for this is shown as Equation (4).

$$
\sigma^{2}(\tau) \cong \frac{1}{2 \tau^{2}(M-2 L)} \sum_{K=1}^{M-2 L}\left(\theta_{k+2 L}-2 \theta_{k+L}+\theta_{k}\right)^{2}
$$

\subsubsection{Noise Parameter Identification}

To obtain Noise Parameters for Gyroscope, an equation for a relation between AV and PSD should be used.

$$
\sigma_{\Omega}^{2}(\tau)=4 \int_{0}^{\infty} S_{\Omega}(f) \frac{\sin ^{4} \pi f \tau}{(\pi f \tau)^{2}} d f
$$

\subsubsection{Angle Random Walk (ARW)}

PSD for ARW is shown as Equation (6).

$$
S_{\Omega}(f)=N^{2}
$$

Substituting Equation (6) into Equation (5) can be shown as Equation (7). 


$$
\sigma^{2}(\tau)=\frac{N^{2}}{\tau}
$$

\subsubsection{Rate Random Walk (RRW)}

PSD for RRW is shown as Equation (8).

$$
S_{\Omega}(f)=\left(\frac{K}{2 \pi}\right)^{2} \frac{1}{f^{2}}
$$

Substituting Equation (8) into Equation (5) can be shown as Equation (9).

$$
\sigma^{2}(\tau)=\frac{K^{2} \tau}{3}
$$

\subsubsection{Bias Instability (BI)}

PSD for BI is shown as Equation (10).

$$
S_{\Omega}(f)=\left\{\begin{array}{c}
\left(\frac{B^{2}}{2 \pi}\right) \frac{1}{f}: f \leq f_{0} \\
0 \quad: f>f_{0}
\end{array}\right.
$$

Substituting Equation (10) into Equation (5) can be shown as Equation (11) and simplified as shown in Equation (12).

$$
\begin{gathered}
\sigma^{2}(\tau)=\frac{2 B^{2}}{\pi}\left[\ln 2-\frac{\sin ^{3} x}{2 x^{2}}(\sin x+\quad 4 x \cos x)+C i(2 x)-C i(4 x)\right] \\
\sigma^{2}(\tau)=\frac{B^{2} 2 \ln 2}{\pi} \\
B=\text { bias instability coefficient } \\
f_{0}=\text { cut }- \text { off frequency } \\
C_{i}=\text { cosine }- \text { integral function } \\
x=\pi f_{0} \tau
\end{gathered}
$$

\subsubsection{Experimental Design and Analysis}

The target sensor in this study is Invensense AM-GYRO VO2(2-Axis), and Full-Scale Range is $\pm 2000 \%$ and Communication Protocol is Analog type. Data processing was performed using dSPACE MicroLabBox and Matlab. Fig $7-8$ shows the target sensor and MicroLabBox.

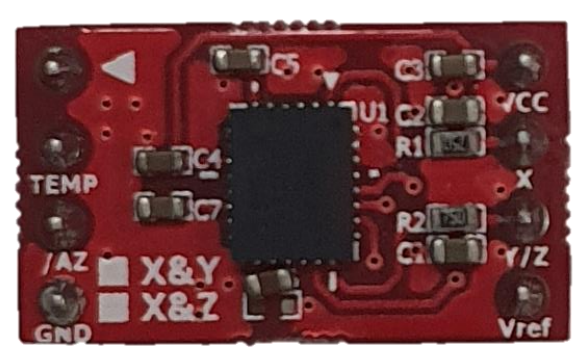

Fig 7.

AM-GYRO V02

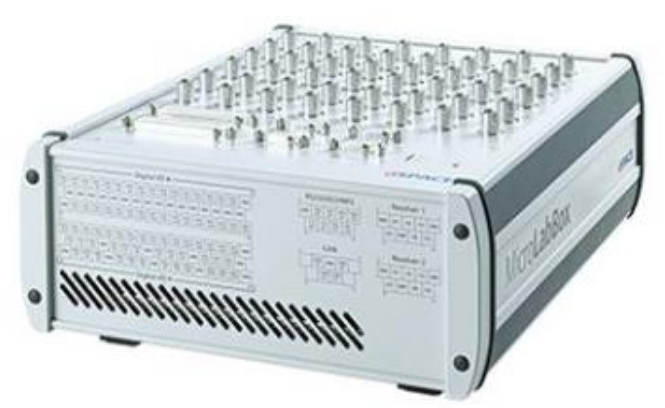

Fig 8. MicroLabBox

Table 2 briefly summarizes the equations derived through AV.

Data was extracted at $1000 \mathrm{~Hz}$ for about 6 hours and 40 minutes, and AV was applied. Fig 9-10 shows the graphs as a result of Allan Variance, and Table 3 shows the derived data

Table 2. Allan Variance and Common Noise 


\begin{tabular}{|c|c|c|c|c|c|c|}
\hline Noise & $\begin{array}{c}\text { Para } \\
\text { meter }\end{array}$ & $\begin{array}{c}\text { Power Spectral } \\
\text { Density }\end{array}$ & $\begin{array}{c}\text { Allan Standard } \\
\text { deviation }\end{array}$ & $\begin{array}{c}\text { Slope of } \\
\text { ADF }\end{array}$ & $\begin{array}{c}\text { Noise } \\
\text { coefficient }\end{array}$ & Unit \\
\hline $\begin{array}{c}\text { Angle } \\
\text { Random } \\
\text { Walk }\end{array}$ & $\mathrm{N}$ & $N^{2}$ & $\sigma^{2}(\tau)=\frac{N^{2}}{\tau}$ & $-1 / 2$ & $\sigma(1)$ & $\frac{\mathrm{deg}}{\sqrt{h}}$ \\
\hline $\begin{array}{c}\text { Rate } \\
\text { Random } \\
\text { Walk }\end{array}$ & $\mathrm{K}$ & $\left(\frac{K}{2 \pi}\right)^{2} \frac{1}{f^{2}}$ & $\sigma^{2}(\tau)=\frac{K^{2} \tau}{3}$ & $1 / 2$ & $\sigma(3)$ & $\frac{\mathrm{deg} / \mathrm{h}}{\sqrt{h}}$ \\
\hline $\begin{array}{c}\text { Bias } \\
\text { Stability }\end{array}$ & $\mathrm{B}$ & $\left\{\left(\frac{B^{2}}{2 \pi}\right) \frac{1}{f}: f \leq f_{0}\right.$ \\
0 & $: f>f_{0}$ & $\begin{array}{c}\sigma^{2}(\tau) \\
=\frac{B^{2} 2 \ln 2}{\pi}\end{array}$ & 0 & $\sigma(i)$ & $\frac{d e g}{h}$ \\
\hline
\end{tabular}

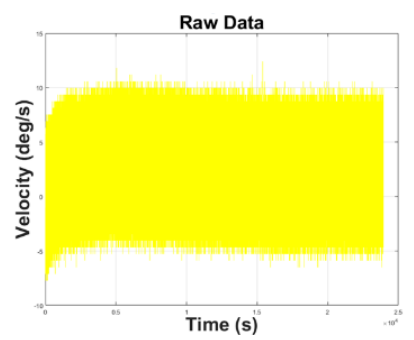

(a) Gyroscope Raw Data

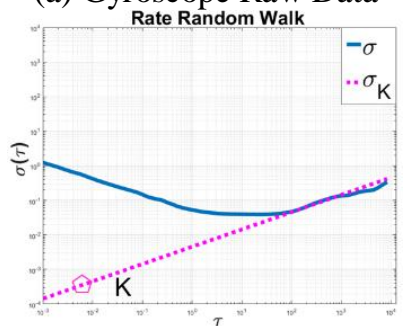

(d) Rate Random Walk

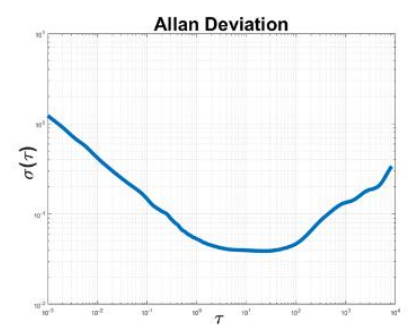

(b) Allan Variance

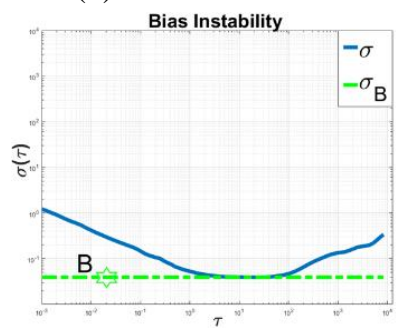

(e) Bias Instability

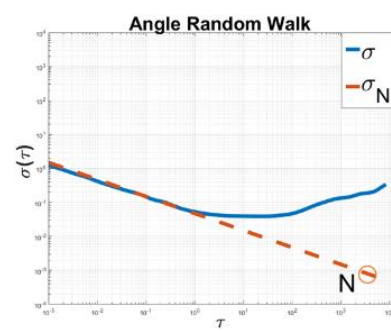

(c) Angle Random Walk

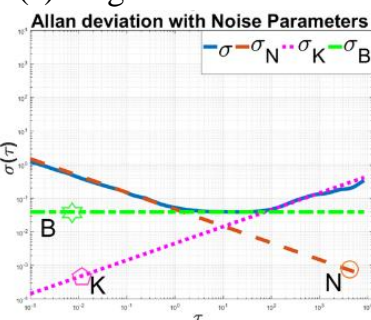

(f) Noise Parameters

Fig 9. AM-GYRO V02(X-Axis)

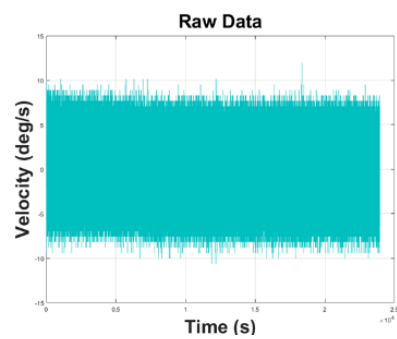

(a) Gyroscope Raw Data

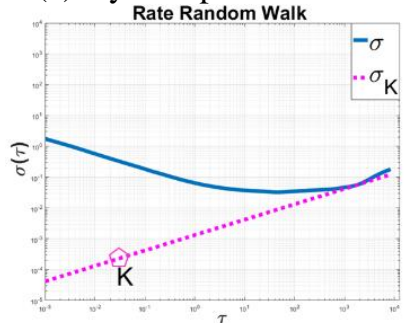

(d) Rate Random Walk

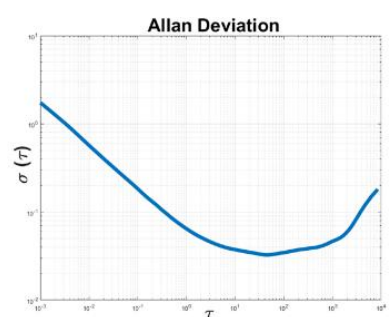

(b) Allan Variance Bias Instability

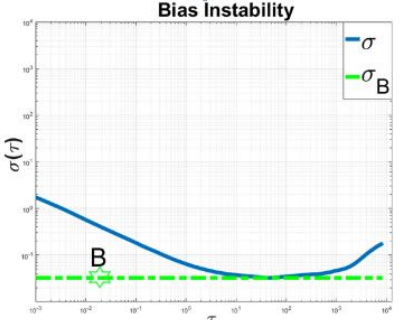

(e) Bias Instability

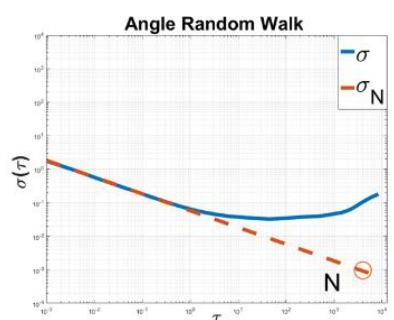

(c) Angle Random Walk

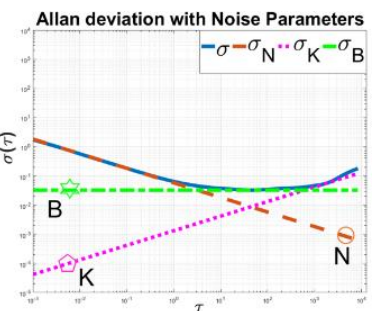

(f) Noise Parameters

Fig 10. AM-GYRO V02(Y-Axis)

Table 3. Axis Error Coefficient 


\begin{tabular}{|c|c|c|}
\hline \multicolumn{3}{|c|}{ AM-GYRO V02 } \\
\hline Noise & X-Axis & Y-Axis \\
\hline Angle Random Walk(N) & 0.0079 & 0.0023 \\
\hline Rate Random Walk(K) & 0.0472 & 0.0578 \\
\hline Bias Stability(B) & 0.0587 & 0.0490 \\
\hline
\end{tabular}

The graph (a) is the raw data of the gyroscope, and (b)-(e) is the graph of ARW, RRW, BI respectively. For ARW, expression (7) and Slope of ADF are -1/2 applied. Expression (9) and Slope of ADF 1/2, BI (12) and Slope of ADF 0 are applied. (f) expresses the whole graph.

\subsection{Optimal Kalman Filter}

As Kalman Filter is widely used in a navigation system, this study was also conducted based on Kalman Filter modeling. Based on system model, KF makes a prediction of updated state and error covariance and calculates a new estimation through compensation for the difference between the predicted value and the measured value [10]. Fig 11 shows the algorithm of Kalman Filter. Gyroscope equation is shown as Equation (13).

$$
\begin{aligned}
& \quad \omega_{g}=\omega+b+n_{a}, \quad b=n_{b} \\
& \omega_{g}=\text { Output Velocity } \\
& \omega=\text { True Velocity } \\
& n_{a}=A R W, \quad b=n_{b}=R R W
\end{aligned}
$$

(x)-1 and $P_{k-1}$

Fig 11. Kalman Filter Algorithm

\begin{tabular}{|} 
Time Update[Predict] \\
\hline 1) Project the State Ahead \\
$\qquad \hat{x}_{\bar{k}}=F \hat{x}_{k-1}$ \\
2) Project the Error Covariance Ahead \\
$\qquad P_{\bar{k}}=F P_{k-1} F^{T}+Q$ \\
\hline
\end{tabular}

\section{Measurement Update[Correct]}

3) Compute the Kalman gain

$$
K_{k}=P_{\bar{K}} H^{T}\left(H P_{\bar{K}} H^{T}+R\right)^{-1}
$$

4) Update Estimate with Measurement $z_{k}$

$$
\hat{x}_{k}=\hat{x}_{\bar{k}}+K_{k}\left(z_{k}-H \hat{x}_{\bar{k}}\right)
$$

5) Update the Error Covariance

$$
P_{K}=P_{\bar{k}}-K_{k} H P_{\bar{k}}
$$

2 Gyroscopes were configured in a serial array, and the angular velocity $\omega$ was extracted as a filtering state to improve the accuracy of sensors. Kalman Filter's state equation and measurement equation were set up as shown in Equation (14).

$$
\left\{\begin{array}{c}
X=\left[b_{1}, b_{2}, \omega\right]^{T} \\
\dot{X}(t)=F X(t)+G \omega(t) \\
Z(t)=H X(t)+B v(t)
\end{array}\right.
$$

$\dot{X}(t)$ is composed of State Vector, and $v(t)$ and $\omega(t)$ are measurement noise and process noise, respectively. Considering the Random Noise of Gyroscope, $\omega(t)=\left[n_{b 1}, n_{b 2}, n_{\omega}\right]^{T}$ and $v(t)=\left[n_{a 1}, n_{a 2}\right]^{T}$ were set. $n_{a}$ is $\mathrm{ARW}$, and $n_{b}$ is RRW. The two noises in Equation (15) satisfy the conditions of the equation in Equation (16).

$$
\left\{\begin{array}{l}
\omega(t)=\left[n_{b 1}, n_{b 2}, n_{\omega}\right]^{T} \\
v(t)=\left[n_{a 1}, n_{a 2}\right]^{T}
\end{array}\right.
$$




$$
\left\{\begin{array}{l}
E\left[\omega_{k}\right]=0, \operatorname{Cov}\left[\omega_{k}, \omega_{j}\right]=E\left[\omega_{k} \omega_{j}^{T}\right]=Q_{k} \delta_{k j} \\
E\left[v_{k}\right]=0, \operatorname{Cov}\left[v_{k}, v_{j}\right]=E\left[v_{k} v_{j}^{T}\right]=R_{k} \delta_{k j} \\
\operatorname{Cov}\left[\omega_{k}, v_{j}\right]=E\left[\omega_{k} v_{j}^{T}\right]=0
\end{array}\right.
$$

$R_{k}$ and $Q_{k}$ of Equation (17) are covariance matrices of measurement noise and system noise, respectively.

$$
Q_{k}=\left[\begin{array}{cc}
Q_{b} & 0 \\
0 & Q_{\omega}
\end{array}\right] \quad R_{k}=\left[Q_{a}\right]
$$

$\mathrm{Q}_{\mathrm{b}}$ and $\mathrm{Q}_{\mathrm{a}}$ are RRW Vector $n_{b}$ and ARW Vector $n_{a}$, respectively.

\section{Experimental Method and Result}

\subsection{Experiment Method}

In this study, the experiment is performed as follows, as shown in Fig 12.

Step 1: Mount two Gyroscopes arranged in series on Rate Table, and connect them to Power Supply

Step 2: Build data reception modeling using Matlab Simulink, and apply it to MicroLabBox

Step 3: Connect the Analog signal of Gyroscope to MicroLabBox, and extract the output value

Step 4: Apply Gyroscope's output value to Matlab Simulink's KF modeling

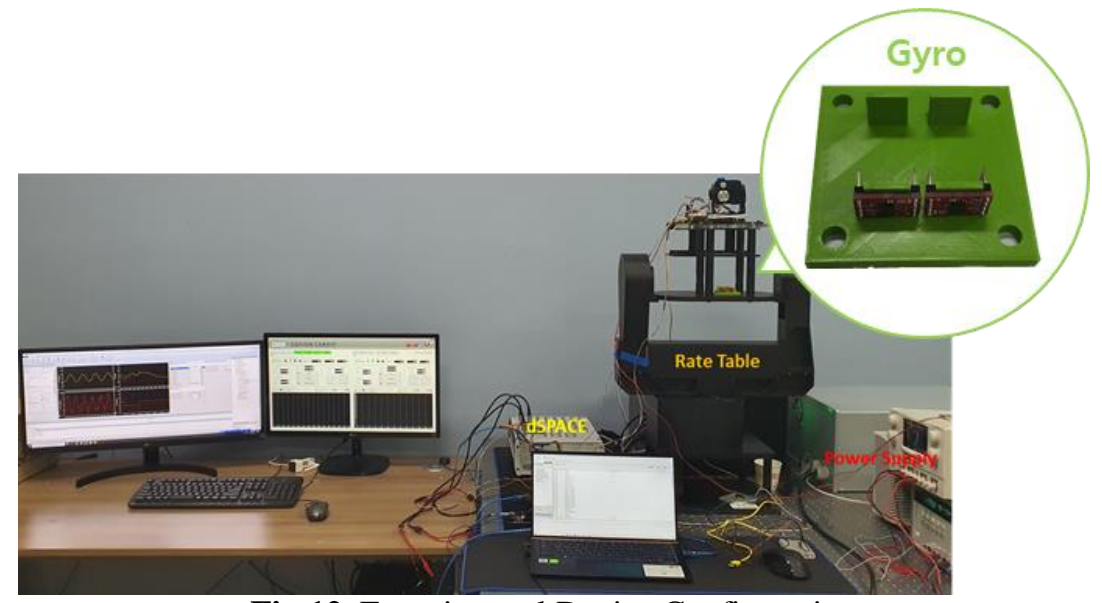

Fig 12. Experimental Device Configuration

\subsection{Experiment Result}

The experiment was conducted with Pan-Axis (Gyroscope X-Axis) and Tilt-Axis (Gyroscope Y-Axis), using the Rate Table at a speed of $30 \mathrm{deg} / \mathrm{sec}$ and a position of $\pm \mathbf{3 0}{ }^{\circ}$. Gyroscope Raw data1 and Raw data2 were extracted using the offset when receiving data. Data was extracted by applying Raw Data 1, 2 to Kalman Filter and Lowpass Filter. KF modeling is the result of this study, and Lowpass Filter produced the optimal value through System Identification and Parameter Study. Fig 13-14 shows Raw Data graphs, a comparative graph of $\mathrm{KF}$ and Raw Data, and a comparison graph of $\mathrm{KF}$ and Lowpass Filter
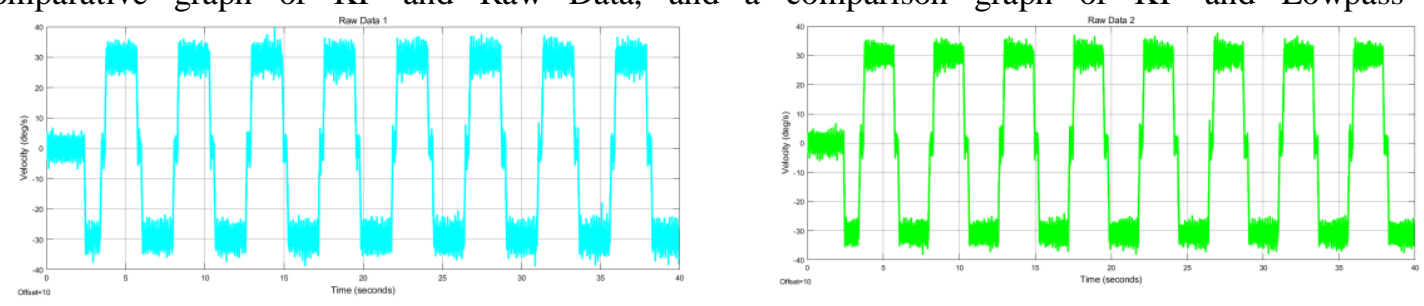

(a) Raw Data 


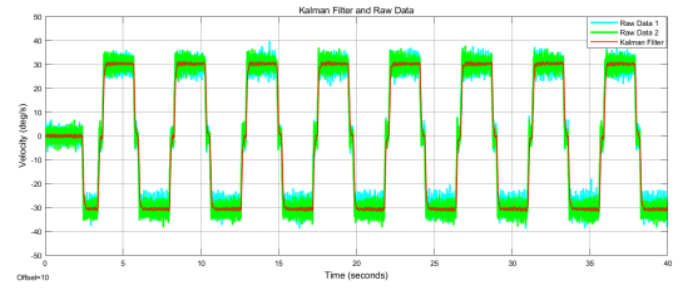

(b) Kalman Filter and Raw Data

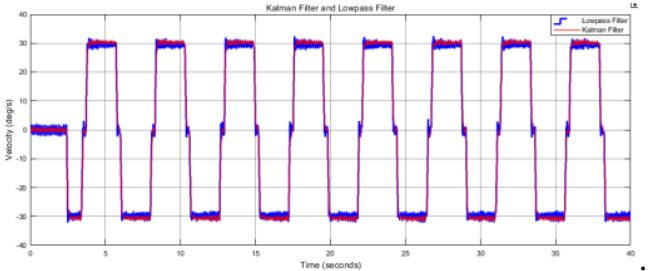

(c) Kalman Filter and Lowpass Filter

Fig 13. AM-GYRO V02[X-Axis]
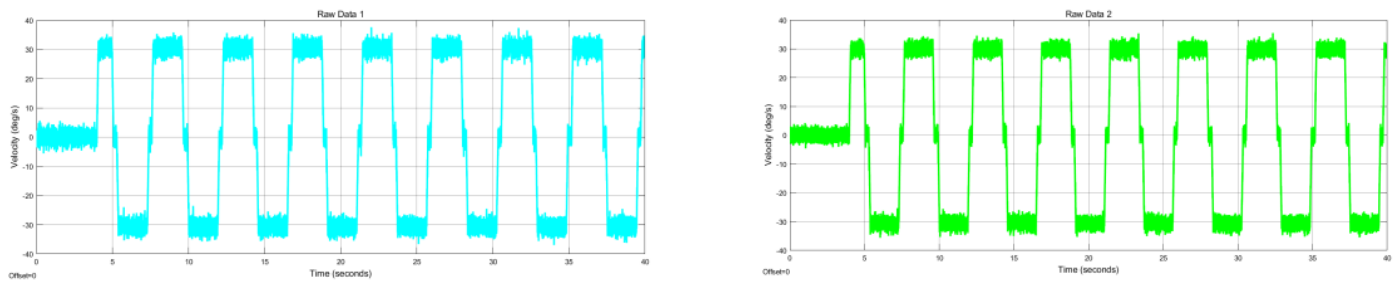

(a) Raw Data

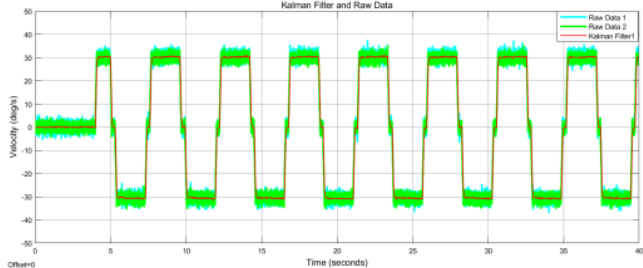

(b) Kalman Filter and Raw Data

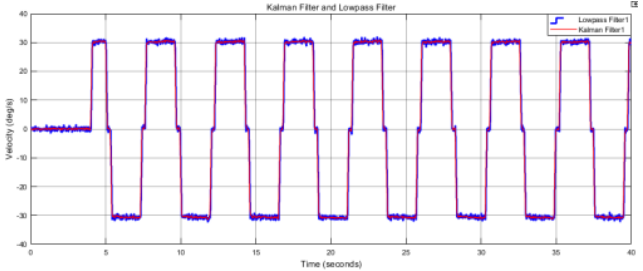

(c) Kalman Filter and Lowpass Filter

Fig 14. AM-GYRO V02[X-Axis]

As a result of comparing the output value when Gyroscope's Raw Data and Lowpass Filter and KF are applied, it is better to track and reduce noises than a single sensor when Kalman Filter is applied to two Gyroscopes. Comparing the lowpass filter applied to the 2-Axis Small Gimbal currently under development with the KF output value of this study, it can be seen that the KF filter has an advantage in reducing noise.

\section{Conclusion}

In this study, an integrated MEMS Gyroscope was examined to improve the accuracy of MEMS sensors to be used in a 2-Axis Small Gimbal. Noise characteristics were analyzed through AV of AM-GYRO V02 sensor, and an experiment was conducted by applying this result to Kalman Filter. It has proven that the method using multi-sensors is very effective in improving accuracy rather than using a single sensor, and it is easy to deal with problems caused by sensor failure. A follow-up study is planning to add the number of sensors and conduct an experiment by applying it to the Kalman Filter developed in this study in real time. If the experiment is sufficiently performed, it will look into the applicability to a 2-Axis Small Gimbal that is currently under development by arranging multi-sensors on one expansion board.

\section{Acknowledgment}

This study is a research paper supported by the 2019 Graduate Student Research Fund Project from the Industry-Academic Cooperation Foundation, Hanseo University.

\section{References}

1. L. Xue, C. Y. Jiang, H. L. Chang, Y. Yang, W. Qin, and W. Z. Yuan, (2012) "A novel Kalman filter for combining outputs of MEMS gyroscope array," ELSEVIER Measurement, 45(4), 745-754. DOI:10.1016/j.measurement.2011.12.016.

2. M. L. Quang, W. J. Thomas, C. Ronald, and B. Darrin, (2004) "Enhancing MEMS Sensors Accuracy Via Random Noise Characterization and Calibration," SPIE 5403, DOI :10.1117/12.538257.

3. M. A. Weiss, D. W. Allan, D. D. Davis, and J. Levine, (1992) "Smart clock: A new time," IEEE 41(6), 915-918. DOI: 10.1109 / 19.199433.

4. H. L. Chang, L. Xue, W. Qin, G. M. Yuan, and W. Z. Yuan, (2008) "An Integrated MEMS Gyroscope Array with Higher Accuracy Output,” MDPI sensors 8(4), 2886-2899. DOI: 10.3390 / s8042886. 
5. C. Babu, R. K. Karri, and M. S. Nisha, (2018) “Sensor Data Fusion Using Kalman Filter,” IEEE 29-36. DOI: 10.1109 / ICDI3C.2018.00015.

6. Q. Gan, C. J. Harris, (2001) "Comparison of Two Measurement Fusion Methods for Kalman-FilterBased Multisensor Data Fusion,” IEEE 37(1), 273-279. DOI: 10.1109/7.913685.

7. S. H. Choi, C. W. Kang, and C. G. Park, (2014) "Calibration and noise characteristic analysis of IMU for cube satellite SNUSAT-1,” The Korean Society for Aeronautical \& Space Sciences 365-368.

8. X. Y. Dai, Z. G. Chen, and X. Xie, (2014) "The gyro random walk analysis based on Allan variance," Trans Tech Publications 668-669, 953-956. DOI: 10.4028/ www.scientific.net/AMM.668-669.953.

9. K. Nirmal, A. G. Sreejith, J. Mathew, M. Sarpotdar, A. Suresh, A. Prakash, M. Safonova, and J. Murthy, (2016) "Noise modeling and analysis of an IMU-based attitude sensor: improvement of performance by filtering and sensor fusion," SPIE. DOI: /10.1117/12.2234255.

10. S. P. Kim, (2010) Kalman Filter's Understanding. Ajin, Seoul. 